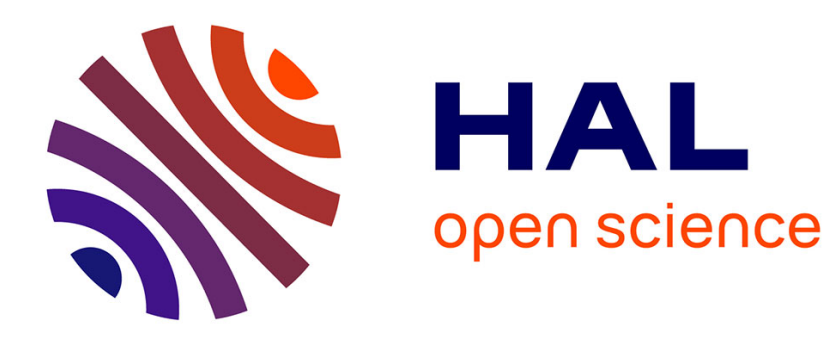

\title{
Deep Learning in Alternate Reality
}

Rufin Vanrullen

\section{To cite this version:}

Rufin Vanrullen. Deep Learning in Alternate Reality. Nature Human Behaviour, 2021, 6 (1), pp.27-28. hal-03452794

\section{HAL Id: hal-03452794 \\ https://hal.science/hal-03452794}

Submitted on 27 Nov 2021

HAL is a multi-disciplinary open access archive for the deposit and dissemination of scientific research documents, whether they are published or not. The documents may come from teaching and research institutions in France or abroad, or from public or private research centers.
L'archive ouverte pluridisciplinaire HAL, est destinée au dépôt et à la diffusion de documents scientifiques de niveau recherche, publiés ou non, émanant des établissements d'enseignement et de recherche français ou étrangers, des laboratoires publics ou privés. 


\section{Cognitive Science}

\section{Deep Learning in Alternate Reality}

Rufin VanRullen ${ }^{1,2}$

1. CerCo, Centre de Recherche Cerveau et Cognition, CNRS UMR 5549, 31052 Toulouse, France

2. ANITI, Artificial and Natural Intelligence Toulouse Institute, Université de Toulouse, 31062 Toulouse, France

Correspondence to Rufin VanRullen: rufin.vanrullen@cnrs.fr

Similarities and differences between deep learning models and primate vision have been the focus of recent research. Audition is, comparatively, less studied. A new report describes the emergence of human-like auditory perception in a deep neural network, and suggests a promising way to relate perceptual behavior to specific aspects of the environment.

Much of Modern Al (Artificial Intelligence) hinges on Deep Learning: neural networks comprising many layers, trained on large datasets using gradient descent optimization methods. These networks are widely used in computer vision, and often surpass the visual abilities of human subjects. A body of recent research has pointed out similarities and differences between such deep neural networks and the primate visual system [1, 2], but comparable auditory studies remained scarce [3, 4]. A new article by Francl and McDermott [5] fills this gap, by revealing how properties of human audition can emerge in deep networks, and how such properties are causally dependent on features of the natural environment.

Francl and McDermott implemented a family of deep convolutional neural networks receiving realistic auditory inputs: simulated natural sounds rendered in a virtual acoustic environment including simulated rooms with background noises, a simulated head and torso, simulated outer ears (pinnae) and a simulated cochlea. The resulting inputs were similar to the range of natural audio signals that enter the human auditory system, except for one crucial difference: they were entirely under the control of the experimenters, who could manipulate the type and 3D position of audio sources, in addition to various properties of the environment (background noises, room structure and reverberation properties, etc.). Because of this, the authors could build a large-scale dataset of labelled examples for training their networks; the training objective was to classify the exact position (azimuth, elevation) of target sources. Through alternating steps of model selection (identifying promising network hyper-parameters such as the number of layers, channels, or the kernel size of the convolutions) and model training with backpropagation, they converged on a set of deep neural networks that could localize sounds, on a separate set of test stimuli, with comparable accuracy to human subjects.

Using a virtual world not only allowed the authors to construct the dataset required for supervised training, but it also gave them fine-grained control over every feature of the environment. This way, they could recreate various experimental conditions (e.g., simulating multiple sources with time lags, a room with no echo, or a modified ear shape). They tested their trained model on a battery of simulated psychophysical tests normally applied to human participants. These tests included: sensitivity to inter-aural time and level differences; azimuthal localization of broadband sounds, and its bandwidth dependence; dependence of elevation judgments on ear-specific cues, spectral smoothing or spectral frequency; localization of multiple simultaneous sources; precedence effect (i.e., a bias towards the location of the first of two near- 
simultaneous targets). Systematically, the model behaved like human listeners, even though it had never been trained on-or even experienced-these experimental conditions before.

This agreement between human and model behavior is already remarkable; but the truly groundbreaking part comes next. The authors again took advantage of their virtual environment, this time not to recreate existing stimuli or experimental conditions, but rather to explore new, physically implausible environments-in other words, alternate realities. In one such reality (the "anechoic" world), they cancelled all sound reverberations. In another (the "noiseless" world), they turned off all background noises. In yet another environment, they replaced all natural sounds with narrowband noise bursts (the "unnatural sounds" world). The deep neural network was retrained from scratch to localize sounds in each of these alternate worlds. Subsequently, they applied the same battery of psychophysical tests to each version of the model. Compared to the model trained under naturalistic conditions (and to human participants), the one trained in a noiseless world localized sounds with abnormal bandwidth dependence. Training with unnatural sounds altered elevation sensitivity, particularly its reliance on high-frequency cues and on the specific ear shape. Finally, training in the anechoic world resulted in impaired sensitivity to interaural time differences, and an absence of the "precedence effect". The latter had long been hypothesized to arise in response to environmental reverberations, but this had been difficult to confirm. Testing a system trained in an alternate anechoic reality elegantly and unambiguously establishes this causal relation: without reverberation, there is no precedence effect. Of course, extending this compelling conclusion from a deep network to the human brain, ultimately, requires a leap of faith in the model's completeness.

Fifty years ago, scientists used to rear animals in altered environments to understand the relation between ecological sensory stimulations, behavior and brain functional networks, and tease apart innate from environmental factors. For instance, kittens reared in a world made only of horizontal and vertical stripes lacked sensitivity to oblique orientations [6], while those reared in stroboscopic illumination could not develop proper motion-selective responses [7]. Now, similar causal links between environmental features and behavioral properties can easily be established in deep neural networks trained in virtual environments. Thus, Al training in an alternate reality can complement animal research, or serve as a pilot exploration prior to launching costly animal studies.

Deep learning has emerged in the last decade as an essential technique for neuroscience and behavioral research [8]. One can implement theoretical principles in deep neural networks, and verify that they produce the expected behavior. One can lesion the networks or ablate some of their components to assess which aspect of the theory is crucial. But importantly, as highlighted here, the combination of deep learning with simulations of virtual worlds presents an additional opportunity - to selectively alter any property of the environment. Training deep networks in such alternate realities thus offers an innovative, invaluable tool to establish true causal links between features of the natural environment and behavior. Beyond the new auditory study by Francl and McDermott, a few sparse examples of this "alternate reality" approach already exist in vision. For instance, visual networks trained in increasingly noisy environments tend to increasingly rely on feedback processes [9]. Recurrent networks that can replicate human behavior in illusory contour perception lose this ability when they are pretrained in an alternate world that includes only geometric shapes, without natural scene statistics [10]. In the near future, it is both likely

and desirable that this approach should be extended to numerous other behavioral functions and sensory modalities, with as many handcrafted alternate realities as necessary. The limit is our imagination.

\section{References}

1. Kriegeskorte, N. (2015), Deep neural networks: A new framework for modeling biological vision and brain information processing. Annual Review of Vision Science. 1: p. 417-446.

2. Yamins, D.L. and J.J. DiCarlo (2016), Using goal-driven deep learning models to understand sensory cortex. Nat Neurosci. 19(3): p. 356-65. 
3. Kell, A.J.E., D.L.K. Yamins, E.N. Shook, S.V. Norman-Haignere, and J.H. McDermott (2018), A Task-Optimized Neural Network Replicates Human Auditory Behavior, Predicts Brain Responses, and Reveals a Cortical Processing Hierarchy. Neuron. 98(3): p. 630-644 e16.

4. Huang, N., M. Slaney, and M. Elhilali (2018), Connecting Deep Neural Networks to Physical, Perceptual, and Electrophysiological Auditory Signals. Front Neurosci. 12: p. 532.

5. Francl, A. and J. McDermott (2021), Deep neural network models of sound localization reveal how perception is adapted to real-world environments. Nature Human Behaviour.

6. Hirsch, H.V. and D.N. Spinelli (1970), Visual experience modifies distribution of horizontally and vertically oriented receptive fields in cats. Science. 168(3933): p. 869-71.

7. Cynader, M., N. Berman, and A. Hein (1973), Cats reared in stroboscopic illumination: effects on receptive fields in visual cortex. Proc Natl Acad Sci U S A. 70(5): p. 1353-4.

8. Richards, B.A., T.P. Lillicrap, P. Beaudoin, et al. (2019), A deep learning framework for neuroscience. Nature neuroscience. 22(11): p. 1761-1770.

9. Mittal, S., A. Lamb, A. Goyal, et al. (2020). Learning to combine top-down and bottom-up signals in recurrent neural networks with attention over modules. in International Conference on Machine Learning. PMLR.

10. Pang, Z., C.B. O'May, B. Choksi, and R. VanRullen (2021), Predictive coding feedback results in perceived illusory contours in a recurrent neural network. Neural Networks.

\section{Competing interests: The author declares no competing interests}

